\title{
60 years of metformin use: a glance at the past and a look to the future
}

\author{
Sally M. Marshall ${ }^{1}$
}

Published online: 3 August 2017

(C) Springer-Verlag GmbH Germany 2017

\section{Abbreviations \\ DPP Diabetes Prevention Program \\ DPPOS Diabetes Prevention Program Outcomes Study \\ eGFR Estimated GFR \\ GLP-1 Glucagon-like peptide-1 \\ PCOS Polycystic ovary syndrome \\ SGLT2 Sodium-glucose cotransporter 2 \\ UKPDS UK Prospective Diabetes Study}

This year marks the 60th anniversary of the first clinical use of metformin for diabetes. From small beginnings (and despite a somewhat chequered history), metformin is currently recommended as the first-line oral glucose-lowering agent in most, if not all, clinical guidelines on the management of type 2 diabetes. Perhaps as a reflection of this, metformin was prescribed for $83.6 \%$ of individuals with type 2 diabetes in the UK in 2013 [1]. Meanwhile, in the USA, metformin was the eighth most commonly prescribed drug consistently from 2008 to 2012 [2], the number of prescriptions rising from 51.6 million in 2008 to 61.6 million in 2012. Metformin and gliclazide are the only two oral glucose-lowering agents on the WHO Model List of Essential Medications [3]. How did this drug reach such a commanding position and is such widespread use justified?

If pharmacological therapy is really needed in addition to lifestyle measures, diabetes specialists and people with type 2

Sally M. Marshall

sally.marshall@newcastle.ac.uk

1 Diabetes Research Group, Institute of Cellular Medicine, Faculty of Clinical Medical Sciences, Newcastle University, 4th Floor William Leech Building, Framlington Place, Newcastle upon Tyne NE2 4HH, UK diabetes want medication that is effective at reducing blood glucose levels, easy to take, preferably has once-daily dosing, has no short- or long-term side effects, carries no risk of hypoglycaemia, does not cause weight gain and is affordable worldwide. In addition, the ideal drug would address the underlying pathophysiology of type 2 diabetes and have added value in terms of reducing non-glycaemic risk factors for, and the incidence of, micro- and macrovascular complications of diabetes. How does metformin match up to these demands?

The medication is certainly an effective glucose-lowering agent, generally reducing $\mathrm{HbA}_{1 \mathrm{c}}$ by approximately $10-15 \mathrm{mmol} / \mathrm{mol}(1.0-1.5 \%)$ in type 2 diabetes [4]. However, we are beginning to realise that not everyone responds to the drug equally and that genetic variation may influence individual response [5]. Further, many people with diabetes would argue that it is not easy to take, finding the large tablet size off-putting and the need for more than oncedaily consumption inconvenient. With 60 years of clinical use, we have a good understanding of metformin's short- and longterm side effects. The common gastrointestinal side effects can be minimised by titrating the dose up slowly, taking the medication with/after food and, if necessary, switching to the extended-release preparation. With long-term use, fear of metformin-associated lactic acidosis initially blighted the use of metformin in some countries. However, a recent systematic review of prospective comparator trials and observational cohorts suggests that lactic acidosis is extremely rare, with a similar incidence in people with diabetes taking metformin or other glucose-lowering agents [6]. Indeed, many people have suggested that the contraindication of metformin use in chronic kidney disease stage 3 and beyond (estimated GFR [eGFR] $<60 \mathrm{ml} \mathrm{min}^{-1}[1.73 \mathrm{~m}]^{-2}$ ) is too restrictive. A further meta-analysis, however, supports the cautious use of metformin in individuals with chronic kidney disease with an eGFR

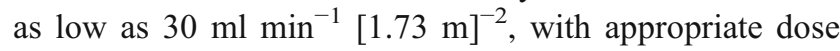


reductions and meticulous ongoing measurement of renal function [7]. However, there is a very small but real risk of lactic acidosis particularly in individuals taking metformin who develop acute kidney injury [8]. Thus, individuals taking metformin and their healthcare providers must remember to omit metformin temporarily during illnesses that may increase the risk of acute kidney injury.

What about metformin and body weight? Clinicians often emphasise the weight-loss effects of metformin, but a systematic review and meta-analysis of placebo-controlled randomised trials suggests that the mean weight loss with metformin is $1.1 \mathrm{~kg}$ [9]. Metformin has a clear advantage over sulfonylureas, which lead to a weight gain of $2-3 \mathrm{~kg}$. However, compared with the glucose-lowering and weightreducing effects of glucagon-like peptide-1 (GLP-1) analogues and sodium-glucose cotransporter 2 (SGLT2) inhibitors, metformin appears not to provide any additional weight benefit [10].

So, overall, in metformin we have a tried and trusted medication that meets some of our criteria for a good medication for diabetes, at least modestly. This is particularly so in comparison with the other class of well-established glucose-lowering agents, the sulfonylureas. However, will the newer classes of glucose-lowering agents succeed in knocking metformin from its lofty pedestal? Currently, these drugs are much more expensive than metformin and their long-term safety is not yet clear, so they are generally recommended as second- or third-line agents for the management of type 2 diabetes. There is a possibility, however, that as data and clinical experience accrue, they will displace metformin and become first-line therapy, at least for some individuals with type 2 diabetes.

The central role of metformin in managing type 2 diabetes is all the more remarkable as, even after 60 years, we still do not fully understand its mechanism of action. That it inhibits hepatic gluconeogenesis is probably beyond doubt, but exactly how it does so is unclear. Whether other actions on the gastrointestinal tract and perhaps even a gut-brain-liver axis are more important is hotly debated [11]. Furthermore, evidence that metformin actually alters the underlying diabetes disease process is lacking. Mainly on the basis of the UK Prospective Diabetes Study (UKPDS) randomised trial and open follow-up results, we believe that metformin reduces the incidence of macrovascular disease at least in obese individuals $[12,13]$, but are uncertain whether this is purely a glucose-lowering effect or whether metformin has other pleomorphic effects. For the GLP-1 analogues and SGLT2 inhibitors, there is preliminary evidence of cardiovascular benefit for individual agents in each class [14-16]. More data from other agents in both classes are awaited.

Metformin is no exception to the current vogue for the repurposing of old drugs for new applications. It is widely used in the management of polycystic ovary syndrome (PCOS), but is this use justified? Further, following the recognition that the risk of some cancers is increased in type 2 diabetes, attention has turned to the effects of glucoselowering agents on cancer. Observational studies have suggested that cancer-related mortality was lower in individuals prescribed metformin compared with those taking insulin or sulfonylureas [17]. Despite several meta-analyses suggesting the contrary (that metformin does not lower cancer risk or mortality $[18,19])$, much effort is currently being devoted to in vitro and in vivo studies exploring potential mechanisms of action and biological effects of this drug on cancer prevention and treatment. Metformin is also increasingly being promoted as an anti-ageing drug [20]. How much substance is in this hype?

To mark this 60th anniversary of the first clinical use of metformin in diabetes, Diabetologia has commissioned a series of articles on many of these controversial and evolving aspects of metformin. In addition to providing a critical review of the current evidence, our authors were asked to look to the future and to speculate where the metformin journey might take us.

Our series begins with an introduction to the drug by Clifford Bailey [21], who outlines its herbal history, tracing it to Galega officinalis, which was first indicated to treat diabetes-associated symptoms in 1772. Bailey describes how it was not until 1957 that a physician named Jean Sterne reawakened interest in metformin. He had noted the fact that flumamine (metformin hydrochloride) was able to lower blood glucose in influenza patients (in whom this drug was being used as an anti-influenza agent). Setting out to investigate the pharmacodynamics of guanidine-based compounds, Sterne 'translated the blood-glucose-lowering potential of metformin into a therapeutic reality'. Motivated by these findings, research between 1960 and 1980 verified the efficacy of this drug for lowering blood glucose, with little evidence for serious safety implications, resulting in this drug eventually being labelled the 'optimal' therapy in type 2 diabetes.

Despite metformin taking the top spot in the hierarchy of glucose-lowering agents, many questions remain regarding the mechanisms of metformin action. Metformin's effects on the liver are widely reported, reducing hepatic gluconeogenesis and improving insulin sensitivity. In their review, Rena and colleagues [22] discuss the impact of metformin on mitochondrial function and AMPK activity in the liver, as well as AMPK-independent effects. Further, the authors highlight a more recently established critical role for the intestine in metformin's actions, with metformin altering glucose metabolism in the gut, suppressing hepatic glucose production via liver-gut-brain crosstalk, and altering the gut microbiome.

Sanchez-Rangel and Inzucchi [23] address the hierarchical place of metformin in clinical therapy. They present data indicating the efficacy of metformin for lowering glucose levels in type 2 diabetes, both as monotherapy and in combination with other agents. In this cohort, the drug also does not appear 
to increase hypoglycaemia risk and is weight-neutral, whilst having potential cardiovascular benefits. The authors do not ignore the adverse effects related to this drug, discussing clinical approaches taken to avoid side effects. Finally, they acknowledge that, although many drug trials have been carried out on a background of metformin therapy, some novel therapies have not been tested head-to-head against metformin. Therefore, they question how this biguanide will fare against new upcoming glucose-lowering agents in the treatment of type 2 diabetes.

In type 1 diabetes, insulin is the first-line therapy. However, despite advances in insulin formulations and modes of delivery, insulin use is associated with increased risk of hypoglycaemia and weight gain, the latter leading to insulin resistance and an escalating insulin dose requirement, higher BP and increased LDL-cholesterol. Therefore, diabetologists and people with type 1 diabetes seek an adjunct to insulin therapy that might improve glycaemic control in type 1 diabetes, without weight gain and its adverse consequences. Could metformin be such a therapy? In their review, Livingstone et al [24] outline evidence of metformin use in type 1 diabetes. Overall, studies suggest that metformin use does not lead to sustained improvements in glycaemic control but reduces insulin dose requirement, weight and, potentially, LDL-cholesterol. The authors also present novel data from the Reducing with Metformin Vascular Adverse Lesions (REMOVAL) study, showing that atherosclerosis progression may also be reduced with metformin use, whilst only transient reductions in $\mathrm{HbA}_{1 \mathrm{c}}$ were observed. Hence, there appears to be potential for this drug to have glucose-independent effects that may be beneficial for those with type 1 diabetes.

Alongside disease management, one of the major goals of therapy is to prevent disease onset. The Diabetes Prevention Program (DPP) is the largest and longest clinical trial of metformin for the prevention of diabetes and, in this review series, Aroda et al [25] present the main metformin-associated findings from the DPP and its follow-on study, the DPP Outcomes Study (DPPOS). Most notably, metformin reduced diabetes incidence by $31 \%$ vs placebo after 2.8 years follow-up, with the reduction in risk (18\%) still being observed over 10 and 15 years post-randomisation. Moreover, favourable effects of metformin on several cardiovascular risk factors were observed. Hence, the findings from the DPP/DPPOS show promise for metformin use for the prevention of type 2 diabetes, with additional benefits extending to its cardiovascular complications.

Griffin et al [26] delve deeper into the association between metformin and cardiovascular disease risk. These authors conducted a meta-analysis of randomised trials reporting cardiovascular outcomes in which the effect of metformin was 'isolated' through comparison with diet, lifestyle or placebo. Risk of all-cause mortality, cardiovascular death, myocardial infarction, stroke and peripheral vascular disease was analysed and all outcomes, except for stroke, favoured metformin; findings suggested that metformin reduces risk of all-cause mortality by up to $16 \%$, but that it may also increase risk of stroke by up to $48 \%$. However, the authors point out that several important limitations (including study bias and small sample size) should be acknowledged when interpreting these findings and that well-designed randomised studies of metformin therapy are needed to enable comparison with newer glucose-lowering drugs (e.g. empagliflozin and liraglutide), for which rigorous cardiovascular endpoint data are available.

For some conditions, metformin stands as a relatively new therapeutic approach. In their review, Sam and Ehrmann [27] discuss the use of metformin for PCOS. In addition to its reproductive manifestations, PCOS also has metabolic consequences, including insulin resistance, impaired glucose tolerance and type 2 diabetes. Metformin was first shown to ameliorate hyperandrogenism in women with PCOS in the 1990s and, although the precise mechanism of action is not fully understood, its use in PCOS is common. Sam and Ehrmann use the small pool of available evidence to outline how metformin acts on PCOS-relevant tissues to reduce hepatic glucose output, increase muscle glucose uptake and regulate androgen production by the ovary. These metformin-induced effects mainly prevent the metabolic consequences of the disease, whilst having only limited effects on reproductive complications. To establish the true potential of metformin in PCOS, however, the authors express the need for large welldesigned prospective trials.

Important insight into metformin use in pregnancy has been gained from studies of its use in PCOS. Metformin has been used in pregnancy for over 40 years. The drug crosses the placental barrier, and the proposed increased lactic acidosis risk and relatively hypoxic fetal environment have raised concerns about its use in pregnancy. However, studies in PCOS suggested that metformin does not increase congenital malformations or miscarriage. In their review, Lindsay and Loeken [28] discuss the use of metformin in gestational diabetes, presenting evidence that metformin has a good safety profile and is effective at reducing rates of severe hypoglycaemia and weight gain in pregnant women. In contrast, however, in pregestational type 2 diabetes the evidence base for metformin use is not strong, but observational evidence suggests increased risk of preeclampsia and perinatal mortality. Evidence of long-term effects of metformin use in the offspring of mothers with gestational diabetes is equivocal for changes in body composition, whilst no differences in BP, and motor, social and linguistic development have been observed. Thus, although there is potential for metformin to act as a suitable therapy in gestational diabetes (but not pregestational diabetes), there is concern with its use because of a paucity of robust data.

Studies have also shown that metformin can reduce diabetes risk in those aged 60 years or over, and also that ageing outcomes, such as frailty and impaired physical and cognitive function, are improved with its use. In their review, Valencia 
et al [29] outline potential mechanisms by which metformin interferes with the ageing process, independent of blood glucose regulation. For example, the effects of metformin on inflammation and reactive oxygen species are thought to reduce DNA damage, whilst its effects on ceramides (which contribute to reduced myoblast numbers in the elderly) may also help to improve tissue health and function. Furthermore, cardio- and neuroprotective roles of metformin, and the impact of metformin on psychological health and cognitive function may also promote healthy ageing and increase lifespan. Valencia et al conclude by outlining several upcoming clinical trials of metformin in ageing that offer an exciting opportunity to discover the true potential of metformin in the ageing population, with or without diabetes.

In his review, Michael Pollak [30] also discusses the nonglycaemic effects of metformin. He suggests that metformin's function may be partly explained by its effect on the human microbiota (e.g. decreased Intestinibacter levels, increased Caenorhabditis elegans lifespan) and gut metabolome (e.g. increased butyrate synthesis), although the precise mechanisms for these changes are yet to be determined. For example, specific microbiota profiles are associated with glucose intolerance, whilst gut-derived metabolites can alter insulin resistance and glycaemic control. Alternatively, metformin may alter gastroenteric mechanisms that influence systemic carbohydrate metabolism, for example, by changing bile acid physiology, GLP-1 levels and duodenal AMPK signalling. Metformin also has immunomodulatory effects via its impact on energy metabolism in immune cells, which may alter immune function. This is thought to result in anti-tumour activities or a reduction in inflammation. These effects of metformin may be relevant in conditions other than diabetes, including cancer.

In line with this, evidence for the association between metformin and reduced cancer incidence and mortality has been provided by epidemiological studies. In this review series, Heckman-Stoddard et al [31] discuss the potential use of metformin for cancer prevention and treatment. Two main routes are proposed to contribute to metformin's anti-neoplastic activity: (1) an indirect route via a reduction in insulin, which may slow tumour proliferation in individuals with hyperinsulinaemia; and (2) reduction of energy consumption in preneoplastic and neoplastic cells via direct action on respiratory Complex I of the electron transport chain. HeckmanStoddard and colleagues go on to discuss findings from clinical trials of metformin in cancer. They demonstrate that, depending on the type of cancer investigated, metformin has a variable impact on cancer-related biomarkers, showing promising results with some cancers but having minimal effects on others. There are many ongoing trials in this research area and we patiently await findings from Phase III trials that will provide important evidence for or against an anti-neoplastic role for metformin.
Regardless of the condition it is being used to treat, the response to metformin therapy varies from person to person. In his review, Jose Florez [32] discusses why heterogeneity in response occurs and how we can predict it. In doing so, the hope is that a personalised approach to metformin therapy may be possible. However, our lack of knowledge of metformin's precise molecular target and mechanism of action make patient stratification difficult. Nonetheless, Florez provides a potential solution: the genetic approach. Candidate gene studies have revealed that mutations in the gene encoding the organic cation transporter 1 (OCT1) are most likely to impact metformin response, compared with other metformin transporter proteins. Findings from genome-wide association studies point towards a role for mutations in ATM and SLC2A2 (the latter encoding GLUT2) in glycaemic response to metformin. However, a personalised approach to metformin therapy is not on the cards just yet, with a need for much more evidence from genetic studies before this hope becomes a reality.

In summary, this review series demonstrates how a drug that could easily have been lost and forgotten following a turbulent start, has not only come to play a leading role in the treatment of type 2 diabetes but also shows promise for the treatment of type 1 diabetes, diabetes in pregnancy, PCOS, ageing and cancer. However, a common theme throughout this series is that robust evidence is lacking for metformin use in many of these conditions, threatening the chance of this drug remaining in the top spot, whilst other newer glucoselowering therapies are quickly being introduced to the market. Nonetheless, at present, metformin remains as one of 'the most efficacious, safe and cost-effective medicines for priority conditions' [3] and, there is a fine chance that in another 60 years' time solid evidence will support its use in diseases other than diabetes.

I hope you will agree that our authors have risen to the challenge we presented to them, and that you find this series informative, stimulating and thought-provoking from both the clinical and scientific perspectives.

\section{References}

1. Sharma M, Nazareth I, Petersen I (2016) Trends in incidence, prevalence and prescribing in type 2 diabetes mellitus between 2000 and 2013 in primary care: a retrospective cohort study. BMJ Open 6: e 010210

2. IMS Institute for Healthcare Informatics. National Prescription Audit December 2012. Available from www.imshealth.com. Accessed 3 May 2017

3. World Health Organization. WHO Model List of Essential Medicines. 19th List. 2015. Available from www.who.int/ medicines/publications/essentialmedicines/EML 2015 FINAL amended_NOV2015.pdf?ua=1. Accessed 3 May 2017

4. DeFronzo RA, Stonehouse AH, Han J, Wintle ME (2010) Relationship of baseline HbA1c and efficacy of current glucose- 
lowering therapies: a meta-analysis of randomized clinical trials. Diabet Med 27:309-317

5. Zhou K, Yee SW, Seiser EL et al (2016) Variation in the glucose transporter gene $S L C 2 A 2$ is associated with glycemic response to metformin. Nat Genet 48:1055-1059

6. Salpeter SR, Greyber E, Pasternack GA, Salpeter EE (2010). Risk of fatal and nonfatal lactic acidosis with metformin use in type 2 diabetes mellitus. Cochrane Database Syst Rev, Issue 4, Art. No.: CD002967. doi: 10.1002/14651858.CD002967.pub4

7. Inzucchi SE, Lipska KJ, Mayo H, Bailey CJ, McGuire DK (2014) Metformin in patients with type 2 diabetes and kidney disease: a systematic review. JAMA 312:2668-2675

8. Connelly PJ, Lonergan M, Soto-Pedre E, Donnelly L, Zhou K, Pearson ER (2017) Acute kidney injury, plasma lactate concentrations and lactic acidosis in metformin users: a GoDarts study. Diabetes Obes Metab doi:10.1111/dom.12978

9. Domecq JP, Prutsky G, Leppin A et al (2015) Drugs commonly associated with weight change: a systematic review and metaanalysis. J Clin Endocrinol Metab 100:363-370

10. Wilding JP, Rajeev SP, DeFronzo RA (2016) Positioning SGLT2 inhibitors/incretin-based therapies in the treatment algorithm. Diabetes Care 39(Suppl 2):S154-S164

11. McCreight LJ, Bailey CJ, Pearson ER (2016) Metformin and the gastrointestinal tract. Diabetologia 59:426-435

12. UK Prospective Diabetes Study (UKPDS) Group (1998) Effect of intensive blood-glucose control with metformin on complications in overweight patients with type 2 diabetes (UKPDS 34). Lancet 352:854-865

13. Holman RR, Paul SK, Bethel MA, Matthews DR, Neil HA (2008) 10-year follow-up of intensive glucose control in type 2 diabetes. N Engl J Med 359:1577-1589

14. Zinman B, Wanner C, Lachin JM et al (2015) Empagliflozin, cardiovascular outcomes, and mortality in type 2 diabetes. N Engl J Med 373:2117-2128

15. Wanner C, Inzucchi SE, Lachin JM et al (2016) Empagliflozin and progression of kidney disease in type 2 diabetes. N Engl J Med 375: 323-334

16. Marso SP, Daniels GH, Brown-Frandsen K et al (2016) Liraglutide and cardiovascular outcomes in type 2 diabetes. N Engl J Med 375: 311-322

17. Bowker SL, Majumdar SR, Veugelers P, Johnson JA (2006) Increased cancer-related mortality for patients with type 2 diabetes who use sulfonylureas or insulin. Diabetes Care 29:254-258
18. Stevens RJ, Ali R, Bankhead CR et al (2012) Cancer outcomes and all-cause mortality in adults allocated to metformin: systematic review and collaborative meta-analysis of randomised clinical trials. Diabetologia 55:2593-2603

19. Farmer RE, Ford D, Forbes HJ et al (2016) Metformin and cancer in type 2 diabetes: a systematic review and comprehensive bias evaluation. Int J Epidemiol 46:728-744

20. Anisimov VN (2013) Metformin: do we finally have an anti-aging drug? Cell Cycle 12:3483-3489

21. Bailey CJ (2017) Metformin: historical overview. Diabetologia doi: 10.1007/s00125-017-4318-z

22. Rena G, Hardie DG, Pearson ER (2017) The mechanisms of action of metformin. Diabetologia doi:10.1007/s00125-017-4342-z

23. Sanchez-Rangel E, Inzucchi SE (2017) Metformin: clinical use in type 2 diabetes. Diabetologia doi:10.1007/s00125-017-4336-x

24. Livingstone R, Boyle JG, Petrie JR, The REMOVAL Study Team (2017). A new perspective on metformin in type 1 diabetes. Diabetologia doi:10.1007/s00125-017-4364-6

25. Aroda VR, Knowler WC, Crandall JP et al (2017). Metformin for diabetes prevention: insights gained from the Diabetes Prevention Program/Diabetes Prevention Program Outcomes Study. Diabetologia doi:10.1007/s00125-017-4361-9

26. Griffin SJ, Leaver JK, Irving GJ (2017) Impact of metformin on cardiovascular disease: a meta-analysis of randomised trials among people with type 2 diabetes. Diabetologia doi:10.1007/s00125-0174337-9

27. Sam S, Ehrmann DA (2017) Metformin therapy for the reproductive and metabolic consequences of polycystic ovary syndrome. Diabetologia doi:10.1007/s00125-017-4306-3

28. Lindsay RS, Loeken MR (2017). Metformin use in pregnancy: promises and uncertainties. Diabetologia doi:10.1007/s00125017-4351-y

29. Valencia WM, Palacio A, Tamariz L, Florez H (2017). Metformin and ageing: improving ageing outcomes beyond glycaemic control. Diabetologia doi:10.1007/s00125-017-4349-5

30. Pollack M (2017). The effects of metformin on gut microbiota and the immune system as research frontiers. Diabetologia doi:10.1007/ s00125-017-4352-x

31. Heckman-Stoddard BM, Decensi A, Sahasrabuddhe VV, Ford L (2017). Repurposing metformin for the prevention of cancer and cancer recurrence. Diabetologia doi:10.1007/s00125-017-4372-6

32. Florez JC (2017) The pharmacogenetics of metformin. Diabetologia doi:10.1007/s00125-017-4335-y 